\title{
Radiation Aware Mobility Paths in Wirelessly Powered Communication Networks
}

\author{
Ioannis Katsidimas \\ Department of Computer Engineering \\ and Informatics, \\ University of Patras, Greece \\ Computer Technology Institute \\ and Press "Diophantus", Greece \\ ikatsidima@ceid.upatras.gr
}

\author{
Emmanouil Kerimakis \\ Department of Computer Engineering \\ and Informatics, \\ University of Patras, Greece \\ kerimakis@ceid.upatras.gr
}

\author{
Sotiris Nikoletseas \\ Department of Computer Engineering \\ and Informatics, \\ University of Patras, Greece \\ Computer Technology Institute \\ and Press "Diophantus", Greece \\ nikole@cti.gr
}

\begin{abstract}
Wireless power transfer (WPT) is an emerging technology that is used in ad hoc networks of battery-powered devices, to deliver energy and keep the network functional. Existing state-of-the-art studies have mainly focused on applying this technology, but the potential risk of electromagnetic radiation (EMR) exposure is really overlooked by them. This still holds for the general case of the $R F$ Wireless Communication networks. Hence, we consider The Minimum Radiation Path Problem of finding the lowest radiation trajectory of an agent moving from a source to a destination point in a network plane.

Different from previous works, we attempt to study (for the first time in the state-of-the-art) path radiation under a more realistic WPT model than the usual one-dimensional models, that have been used in the past and cannot capture interesting superadditive and cancellation effects between distinct electromagnetic sources. In the light of the above, we design and evaluate both an algorithm and a heuristic that achieve different trade-offs between radiation and trajectory length of a moving agent.

Index Terms-Wireless Power Transfer, Vector Model, Electromagnetic Radiation, Mobile agents, Ad-hoc Wireless Networks
\end{abstract}

\section{INTRODUCTION}

Even though Wireless Power Transfer (WPT) has been introduced for a decade now, we still face challenges over this extraordinary technology. Several companies and commercial products have been introduced in the market nowadays, equipping RF harvesting applications and systems that support it. So it is necessary more than ever to study and overpass hardware restrictions by applying efficient algorithmic solutions and take wireless networks to a next level of efficacy and performance.

Since many years, several standardization bodies are interested in radiation regulations that all of these systems should follow. As several research outcomes have shown, radiation exposure may induce long-term health effects. Thus, current systems (both in research and industry) start taking into account the need for EM safety.

Our contribution. While current research works have been invested into radiation safety, most of the models studied in the literature ignore key features of electromagnetic fields. Such models are unable to describe various phenomena occurring in real applications (e.g. super-additive and cancellation effects), resulting in impractical algorithmic approaches. Thus, our contribution in this paper includes the following:
- This is the first work, to the best of our knowledge, that attempts to study radiation aspects in WPT and $\mathrm{RF}$ communication with respect to the vector model [2], which is a new approach for radiation awareness in wireless settings.

- We provide an algorithm and a heuristic that solve the Minimum Radiation Path Problem and are evaluated against another state of the art algorithm.

- Finally, we evaluate the designed mechanisms which outperform the state of the art algorithm and other naive approaches.

Roadmap of the paper. The rest of this paper is organized as follows. Section 2 elaborates on the related works. The network and radiation model are explained in Section 3. Section 4 defines the Minimum Radiation Path Problem definition and includes the proposed algorithms. Finally, in 5 we present the outcomes of the evaluation and in Section 6 the conclusion.

\section{RELATED WORK}

Research efforts in Wireless Communication Networks and WPT technology have already started considering solutions that address Electromagnetic Radiation (EMR) issues.

Regarding the model exploitation, there is a work ( [1]) that algorithmically addresses Power Maximization Problem and make use of the vector model for WPT networks. Generally, the main problem to solve concerning $E M R$, is to maximize the received power while the radiation level remains low ( [3], [4], [13]) and vice versa, in the sense that we try to minimize radiation levels and sufficiently charge the devices ( [12]). In [3], the authors consider the Safe Charging with Adjustable PowEr (SCAPE) problem, where they adjust the power of chargers to maximize the charging utility of devices, while assuring that EMR intensity at any location in the field does not exceed a given threshold. Similarly, [4] tries to maximize the minimum utility of devices by adjusting the power of wireless chargers with no EMR intensity at any location in the field exceeding a given threshold. [6] studies the problem of radiation constrained scheduling of wireless charging tasks. So, given wireless charging tasks with required charging energy and charging deadline for rechargeable devices, schedule the 
power of wireless chargers to maximize the overall effective charging energy for all rechargeable devices. Further minimize the total charging time, while guaranteeing electromagnetic radiation EMR safety.

Research has also been conducted on the fundamental issue of wireless charger placement with electromagnetic radiation (EMR) safety. In [5] the authors propose PESA, a wireless charger placement scheme that guarantees EMR safety for every location on the plane. In [7], a new approach is studied regarding the problem of efficiently charging a set of rechargeable nodes using a set of wireless energy chargers, under the EMR safety constraints. [8] attempts (for the first time from a distributed networking perspective) to investigate the aspect of electromagnetic radiation in modern heterogeneous wireless networks and propose three heuristics which provide low radiation paths while keeping path length low. [9] further investigates the paradigm introduced in [8] and focuses on the fundamental problem of efficient data propagation in wireless sensor networks with low latency. All this is done while maintaining at low levels the radiation cumulated by wireless transmissions. Last but not least, in [11] a statistical method to evaluate risks to the general public due to electromagnetic radiation in wireless networks as well as their performance and quality-of-service is proposed.

In all above works, our goal of finding efficient moving paths which are at the same time radiation aware has not been addressed.

\section{THE RADIATION MODEL}

In a recent paper [2], the authors considered a model for the superposition of electromagnetic fields created by independent wireless energy sources. The model of [2] goes beyond (in fact, it is a generalization of) the one-dimensional abstraction suggested by Friis' formula for the power received by one antenna under idealized conditions given another antenna some distance away. Here, is briefly presented the formulation conducted in [1], regarding the fundamental properties of the superposition of electromagnetic waves from physics. In particular, the electric field created by an energy transmitter $C$, operating at full capacity, at a receiver $r$ at distance $d=\operatorname{dist}(C, r)$ is a 2-dimensional vector given by

$$
\mathbf{E}(C, r) \stackrel{\text { def }}{=} \beta \cdot \frac{1}{d} \cdot e^{-j \frac{2 \pi}{\lambda} d}=\beta \cdot \frac{1}{d} \cdot\left[\begin{array}{c}
\cos \left(\frac{2 \pi}{\lambda} d\right) \\
\sin \left(\frac{2 \pi}{\lambda} d\right)
\end{array}\right],
$$

where $\lambda$ depends on the frequency at which the transmitter operates, and $\beta$ is a constant that depends on the hardware of the transmitter and the environment. ${ }^{1}$

The main point of the power model of [2], which also sets it apart from other (less realistic, but more tractable) models

${ }^{1}$ In fact, the exact formula used in [2] for the electric field is $\mathbf{E}(C, r) \stackrel{\text { def }}{=}$ $\sqrt{\frac{Z_{0} G_{C} P_{C}}{4 \pi d^{2}}} \cdot e^{-j \frac{2 \pi}{\lambda} d}$, where $Z_{0}$ is a physical constant indicating the waveimpedance of a plane wave in free space, $G_{C}$ is the gain and $P_{C}$ is the output power of the transmitter. In this paper, without loss of generality of our algorithmic solutions, we assume that all wireless transmitters and receivers are identical, thus the aforementioned parameters are the same for each charger. in the wireless charging literature, is that the total electric field created by a family of energy transmitters $\mathcal{C}$ at a receiver $r$ is the superposition (vector-sum) of their individual electric fields, that is

$$
\mathbf{E}(\mathcal{C}, r) \stackrel{\text { def }}{=} \sum_{\mathbf{C} \in \mathcal{C}} \mathbf{E}(C, r)
$$

\section{A. Point Radiation}

Regarding the total radiation (power) at the receiver $r$, this is given by

$$
R(\mathcal{C}, r)=\gamma \cdot\|\mathbf{E}(\mathcal{C}, r)\|^{2}
$$

where $\|\cdot\|$ denotes the length (2-norm) of the vector. The constant $\gamma$ depends on the hardware of the transmitter and the hardware of the receiver.

Below we provide an example to give some intuition of the phenomenon and how we try to solve it: Assume there are two transmitters $C_{1}$ and $C_{2}$ placed at points $(0,0)$ and $(2,0)$ in the 2-dimensional plane. First, consider a receiver $r$ placed at $(1,0)$. Assume also, for the sake of clarity that all constants in the above model are set to 1 , i.e. $\lambda=\beta=\gamma=1$. When only one of the two transmitters is operational, the radiation received by $r$ is $R\left(C_{1}, r\right)=R\left(C_{2}, r\right)=\left\|\mathbf{E}\left(C_{1}, r\right)\right\|^{2}=$ $\left\|\mathbf{E}\left(C_{2}, r\right)\right\|^{2}=\left(\frac{1}{\operatorname{dist}\left(C_{1}, r\right)}\right)^{2}=1$. On the other hand, if both transmitters are operational, the radiation received by $r$ is given by equation (2), that is

$$
R\left(\left\{C_{1}, C_{2}\right\}, r\right)=\left\|\mathbf{E}\left(C_{1}, r\right)+\mathbf{E}\left(C_{2}, r\right)\right\|^{2} .
$$

Furthermore, it is not hard to see that since $r$ is equidistant from either $C_{1}$ or $C_{2}$, the vectors $\mathbf{E}\left(C_{1}, r\right)$ and $\mathbf{E}\left(C_{2}, r\right)$ point to the same direction. Therefore, $R\left(\left\{C_{1}, C_{2}\right\}, r\right)=$ $4 R\left(C_{1}, r\right)=2\left(R\left(C_{1}, r\right)+R\left(C_{2}, r\right)\right)=4$. Notice then that the radiation received by $r$ when both transmitters are operational is larger than the sum of the radiations it receives when only one of the transmitters is operational; this is the so-called superadditive effect and is visible in local maxima in the curve shown in Figure 1b.

Second, consider a receiver $r^{\prime}$ placed at $\left(\frac{5}{4}, 0\right)$. Then by equation (1), $\mathbf{E}\left(C_{1}, r^{\prime}\right)=\frac{4}{5} \cdot\left[\begin{array}{l}0 \\ 1\end{array}\right]$, and also $\mathbf{E}\left(C_{2}, r^{\prime}\right)=$ $\frac{4}{3} \cdot\left[\begin{array}{c}0 \\ -1\end{array}\right]$. By equation (2), the radiation received by $r^{\prime}$ when both transmitters are operational is $R\left(\left\{C_{1}, C_{2}\right\}, r^{\prime}\right)=$ $\left(\frac{8}{15}\right)^{2} \approx 0.28$. Notice then that the radiation received by $r^{\prime}$ when both transmitters are operational is much less than $\min \left\{R\left(C_{1}, r^{\prime}\right), R\left(C_{2}, r^{\prime}\right)\right\}=\left(\frac{4}{5}\right)^{2} \approx 0.64$; this is the socalled cancellation effect and is visible in local minima in the curve shown in Figure 1b.

\section{B. Path Radiation}

Let $P$ be a (finite) trajectory inside the plane $A$. Assuming an agent travels on $P$. Instead of calculating the integral of the radiation, we can approximate it by the following sum: Let $d_{x}$ be a very small displacement over $P$ in positions $r_{i}$ and $r_{i+1}$ , then

$$
\mathcal{R}=\sum_{j=i}^{i+1} R\left(\mathcal{C}, r_{j}\right)
$$




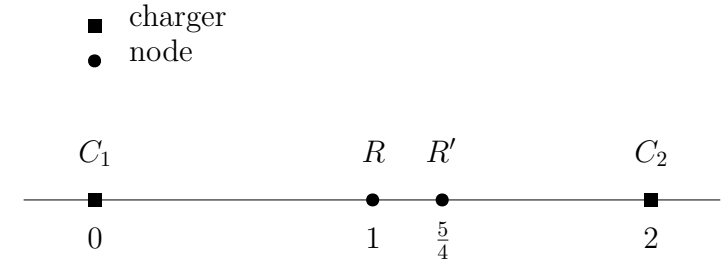

(a) Transmitters' $\left(\mathcal{C}_{1}\right.$ and $\left.\mathcal{C}_{2}\right)$ and agents' $\left(R\right.$ and $R^{\prime}$ ) placement on a straight line at points $(0,0),(0,2)$ and $(0,1),\left(0, \frac{5}{4}\right)$ respectively.

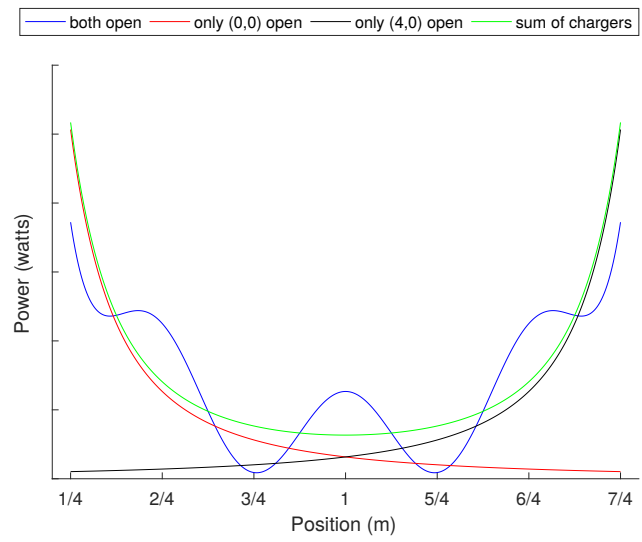

(b) The radiation (power) distribution between the two transmitters placed at points $(0,0),(0,2)$. Different curves represent both different operation states of the transmitters and models.

Figure 1. Example showing the superadditive and cancellation effects.

Eventually, the smaller $d_{x}$ is, the better the approximation is to the actual value.

\section{The Minimum Radiation Path Problem}

Definition IV.1. The Minimum Radiation Path Problem: Given a family of chargers $\mathcal{C}$ and a mobile agent $r$ that wants to travel from point $S$ to point $T$, find a trajectory $P$ from $S$ to $T$ that minimizes the radiation exposure of $\mathrm{r}$ during its travel with respect to travel distance.

We assume that the mobile agent can move at least $\lambda$ distance away from a transmitter. Otherwise, it receives high level of power that does not follow this model as it has also been described in [1].

We propose two algorithms for this problem. In the first algorithm (Surf), the mobile agent can interact with the transmitters in its communication range and exchange information regarding energy sources positions. Hence, the algorithm approaches the points which show low radiation levels, and at the same time guide the agent to the target point. The second, offline algorithm (Graph), is aware of the radiation of the plane and creates a graph containing only low radiation points as vertices and edges, with the radiation and length of the corresponding edge as weight. Then, using a shortest path algorithm, proposes a low radiation path.

\section{A. Algorithms}

In Figure 2 you can see the intensity and patterns of the harvested power depending on different number of transmitters

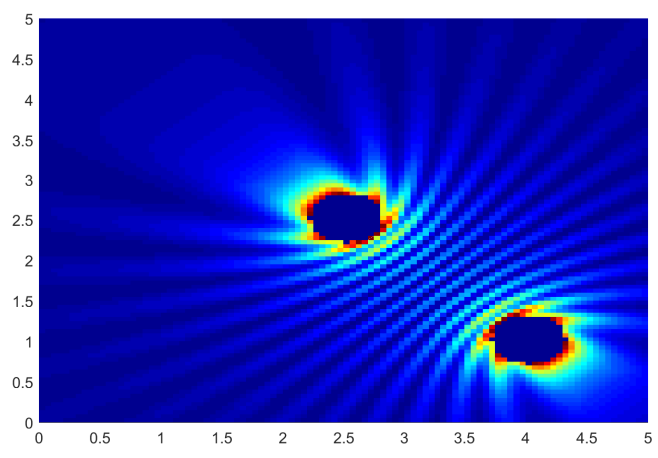

(a) 2 chargers.

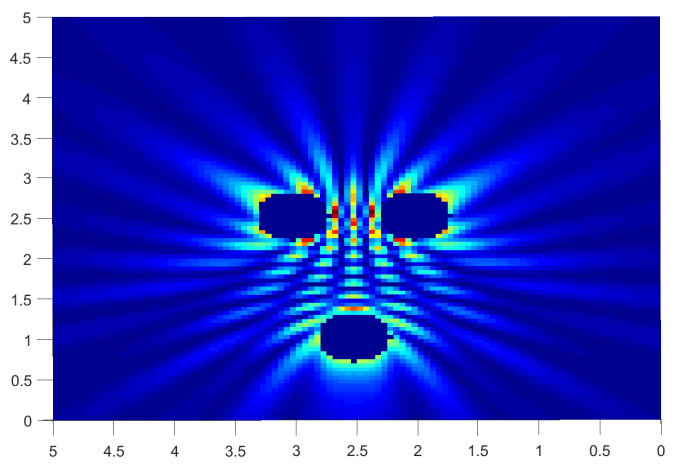

(b) 3 chargers

Figure 2. Power distribution in different number of chargers.

and topologies. It is evidence that there are points close to the transmitters where power drops due to the destructive interference, while instead there are points at distance of transmitters which show high harvested power owing to the constructive interference. Thus, our algorithm SURF, tries to catch a "low radiation wave" and drive the mobile agent at his destination through it.

Surf Algorithm: The algorithm's main goal is to find the points where destructive interference occurs and include them in the path. For two transmitters, these points have the property that the absolute difference of their distances to the two transmitters is an odd multiple of $\lambda / 4$. That means that they are part of the conic section of hyperbola. If $\mathrm{c}$ is half the distance between two chargers, $n=\left[\frac{c}{\lambda / 2}\right\rfloor$ is the number of hyperbolas formed between them. The foci of these hyperbolas are the positions of the two chargers and their center is in the middle of the line segment formed by the chargers. The vertices are placed, on the same line segment, at distance $a_{k}$ from the center equal to odd multiples of $\lambda / 4$. That means $a_{k}=k \cdot \lambda / 4$, where $1 \leq k \leq n$.

The equation that describes them is as follows:

$$
\frac{x_{h}^{2}}{a_{k}^{2}}-\frac{y_{h}^{2}}{b_{k}^{2}}=1
$$

$$
x_{h}=\left(x-x_{c e n}\right) \cdot \cos \theta+\left(y-y_{c e n}\right) \cdot \sin \theta
$$


Table I. Notations

\begin{tabular}{l|l}
\hline Symbol & Meaning \\
\hline$x$ & current position \\
$x^{\prime}$ & new position \\
$S$ & source point \\
$T$ & target point \\
$\mathcal{C}_{x}$ & chargers in communication range at point $x$ \\
$C_{j}$ & charger $j$ \\
$c e n$ & center of circle \\
$p$ & pair chosen \\
$p_{\text {old }}$ & previous pair chosen \\
$H_{i}$ & hyperbola of pair $i$ \\
\hline
\end{tabular}

$$
y_{h}=-\left(x-x_{c e n}\right) \cdot \sin \theta+\left(y-y_{c e n}\right) \cdot \cos \theta
$$

where $\theta=\operatorname{atan} 2\left(\left(y_{C_{2}}-y_{C_{1}}\right),\left(x_{C_{2}}-x_{C_{1}}\right)\right), C_{1}$ is the first charger, $C_{2}$ is the second charger, cen is the center and $\theta$ is the angle between $C_{1}, C_{2}$. With $x_{h}$ and $y_{h}$ we apply the rotation of the hyperbola by angle $\theta$ and the center modification. Note that $b_{k}=c^{2}-a_{k}$.

Therefore, our objective is to avoid the radiation caused by two chargers each time, since they create a low radiation path (hyperbola). Whereas, if we seek to follow points where destructive interference occurs by more than two chargers, these are few and scattered on the plane.

In our algorithm we choose to use only the hyperbolas closest to the center of each pair of chargers $(k=1)$, in order to reduce complexity but also because their radiation is lower, as the distance from the power sources is higher.

Let $x$ be our current position, at first equal to $S$. As long as $x$ is not equal to the target point $\mathrm{T}$, we repeat the following steps. Firstly, we check how many chargers there are in our communication range at $x$ and add them to a set $\mathcal{C}_{x}$.

If $\mathcal{C}_{x}$ is empty, we move one step towards $\mathrm{T}$. If there is only one charger $C_{j}$ in $\mathcal{C}_{x}$, we move on the circle with center the position of $C_{j}$ and radius equal to a radius threshold (slightly smaller than the communication range). Then we check whether the distance of the new point to $\mathrm{T}$ is less than its distance to the center of the circle, and whether $\mathrm{T}$ is inside the circle. If so, we move to $\mathrm{T}$. Lastly, given that the new point is on a tangent line to the circle that crosses $\mathrm{T}$, and $\mathrm{T}$ is outside the circle, we move out of the circle.

In the case of two or more chargers in $\mathcal{C}_{x}$, we create each of the $\left(\begin{array}{c}N \\ 2\end{array}\right)$ possible pairs of chargers. For every pair, we calculate the equation of their hyperbola. Then, we find the projection points of $x$ to the hyperbola and choose the closest one to $x$. The distance between $x$ and the chosen point is calculated (distHypePos). We do the same for $\mathrm{T}$ (distHypeT). Additionally, we take into consideration the mean distance of $x$ to the two chargers (distPos_ij). The pair with the smallest sum including these three parameters, that are multiplied by some weight constants ( $v, w, u$ respectively), is the pair we choose. That means, we select a pair that not only is near to our position but also traversing its hyperbola will bring us closer to T than other pairs' hyperbolas would. In case both chargers of a pair are over or below the line that connects $x$ with $T$ and their distance to this line is higher than

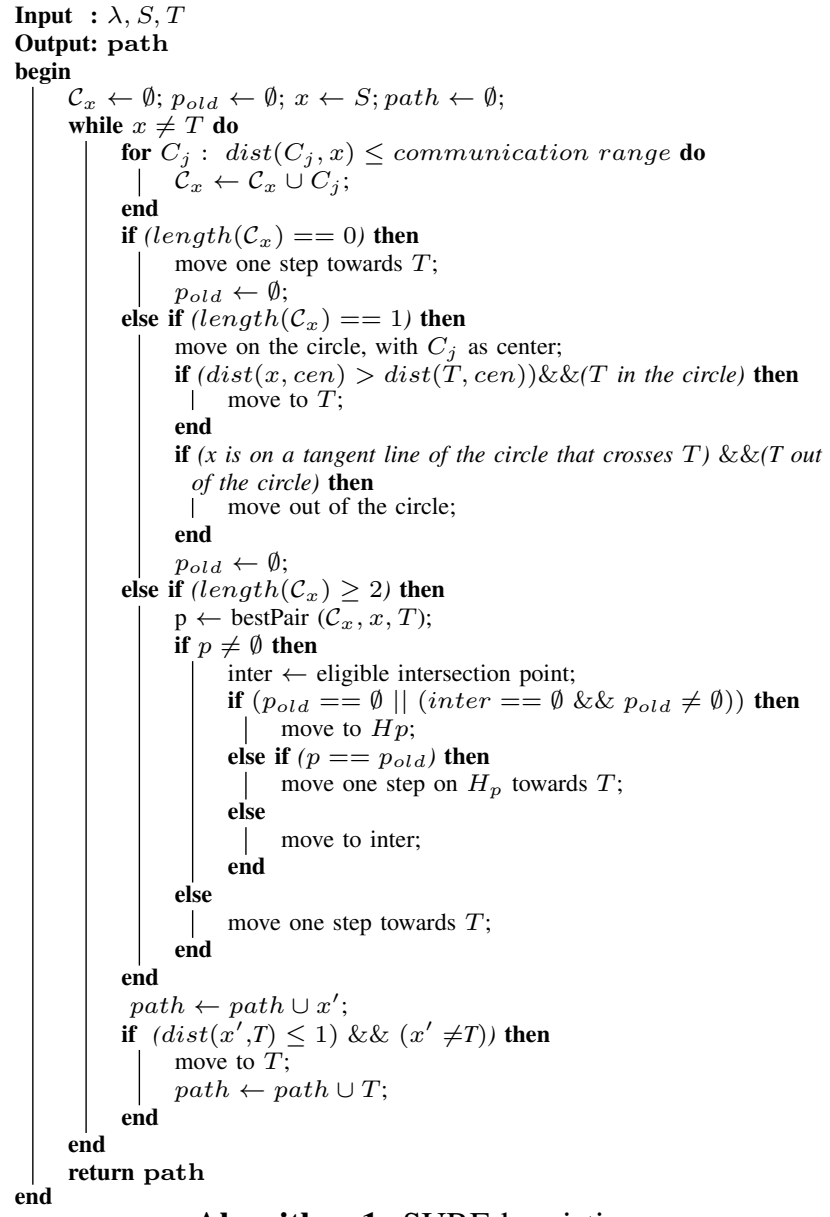

Algorithm 1: SURF heuristic

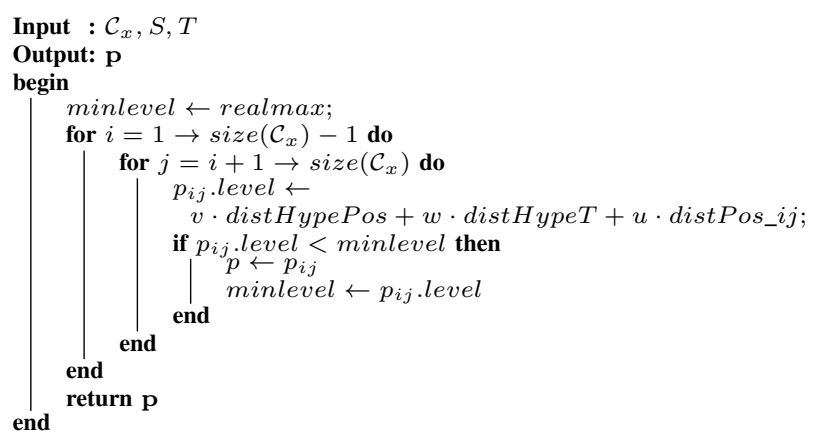

Function bestPair, determines between which pair of chargers the agent will move

$z=$ communication range/2, we ignore them. If no pair is chosen, we walk one step towards $\mathrm{T}$.

Let $p$ be the selected pair and $p_{\text {old }}$ be the previous pair chosen, providing that there is one. Let also, $H_{p}$ and $H_{p_{\text {old }}}$ be their hyperbolas respectively. If we currently are on $H_{p_{\text {old }}}$ and want to move to $H_{p}$, we first check for intersection points between the two. If there are any, we check if their distance to the centers of $H_{p_{\text {old }}}$ and $H_{p}$ is less than $d$ ( $d$ is a parameter depending on the density of the network). If so, we move on $H_{p_{\text {old }}}$ until we find the closest one to our position. Then 
we make a step on $H_{p}$. Unless there are intersection points, we find the closest point, $q$, to our position on $H_{p}$, that is at distance equal to 1 to $H_{p}$ 's center (so that we don't walk on the pair of chargers). In the case $p_{\text {old }}$ is empty, meaning we are not on a hyperbola, we move to the point $q$ described above. Lastly, if $p$ equals $p_{\text {old }}$ we just make one more step on $H_{p}$ towards T. Generally, every time we get onto a new hyperbola, we have to make enough steps on it, so we get past the two chargers. Unless that's true we do not search for a new pair, given we don't find new chargers at distance less than $z$. In addition, we have to notice that we only move on the branch of the hyperbola (each one has two branches) that we chose first.

After any of the described steps, we check whether the distance of the new point to $T$ is less than 1 . If so, we move straight to $T$.

Graph Algorithm: For our second algorithm the creation of a graph $G=(V, E)$ is required. At first we tessellate the $x-y$ axis plane A into $n^{2}$ squares. Then, for every hyperbola $H_{k}$ of the $\left\lfloor\frac{c}{\lambda / 2}\right\rfloor$ (except the ones closest to the chargers) formed between each pair of chargers, we examine at which points they intersect with other hyperbolas. The vertex set $V$ of the graph consists of these intersection points but also of points where the hyperbolas cross the limits of the plane. More formally, if $H_{l}$ is one of the hyperbolas which intersects with $H_{k}$, and $H_{k}(x)=0, H_{l}(x)=0$ are the equations of the two hyperbolas, then the points $p_{\text {int }}$ are the points described above. Given that, the vertex set is $V=\left\{p_{\text {int }}=\left(x_{i n t}, y_{i n t}\right) \mid\left(\left(H_{k}\left(x_{i n t}\right)=y_{i n t}\right) \cap\left(H_{l}\left(x_{i n t}\right)=\right.\right.\right.$ $\left.\left.y_{\text {int }}\right)\right) \cup\left(x_{\text {int }}=\right.$ start $x \cup y_{\text {int }}=$ starty $\cup x_{\text {int }}=$ stopx $\cup y_{\text {int }}=$ stopy)\}, where startx, starty, stopx and stopy are the limits of the plane.

For every hyperbola, we connect with edges all the vertices that belong to it, in order, from end to end, creating this way the edge set, $E$. With this method we save memory compared to other implementations, because the Vertex set contains only the intersection points between the hyperbolas and not all the points of the plane.

After the graph has been created, the problem can be reduced to the problem of finding a minimum weight path between vertices $S$ and $T$. If $S$ or $T$ are not on a vertex, we use the closest vertices to them and then move on a straight line to them. For each edge, we calculate the cumulative radiation $r$ of the respective hyperbola's section and its length $d$. We use the linear combination of these quantities as edge weight. We adjust the weights properly in order to satisfy our constraints about radiation and distance. In this work, we assign weight to each edge $e \in E$ as follows: $w(e)=10 \cdot r(e)+d(e)$. Then we apply Dijkstra's algorithm to find the shortest path.

\section{EVALUATION}

We conclude the presentation of our solutions by demonstrating the performance evaluation which has been conducted in Matlab 2017a environment. We consider a simulation setup

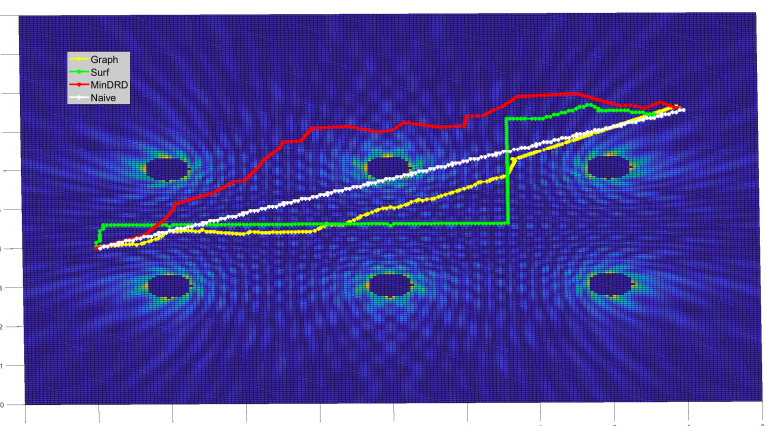

Figure 3. Instance of Surf $\square$, Graph $\square, \operatorname{MinDRD} \square$ and naive $\square$ paths in grid network.

with $\mathrm{n}=6$ chargers $^{2}$ deployed in a $10 \mathrm{~m}^{2}$ network area A, communication range equal to $2 m$ for both grid and random networks and wavelength $\lambda=3 \mathrm{~cm}$. For the Surf algorithm the weights constants, multiplied with the parameters, we used for choosing a pair were $v=1, w=3, u=1$. Also $d=3 \mathrm{~m}$. These weights were chosen after systematic search. For every algorithm, the plane was split into squares of side length equal to 0.05 . We create a probability distribution over these two network topologies by repeating our simulation 100 times for statistical smoothness (the corresponding confidence intervals are presented, verifying a high concentration around the mean value), so that different start and destination points are chosen equiprobably every time for the grid case, while for the random deployment, we consider different topologies. Graph and Surf are going to be compared directly with a naive approach and MinDRD.

MinDRD: The algorithm MinDRD described in [8] also looks for a low radiation path, but it is based on the Friis model. At each step, the moving agent chooses uniformly at random $\mathrm{k}^{3}$ points inside the communication range, each at a different distance $d_{i}$ from the current, $1 \leq i \leq k$. From these $\mathrm{k}$ points, the point $p_{j}$ that minimizes the product of the radiation at its position and its distance to the target point, is selected. Let $C$ be the circle with center the current position and radius the distance $d_{j}$. Also let $p_{d}$ be the point of $C$ that is closest to the target point. The next step point will be in the middle of the minor arc of $C$ created by $p_{i}$ and $p_{d}$. This procedure is repeated until the target point is found in the communication range. Then they move straight to it.

Naive Algorithm: We also create a naive algorithm that simply follows the path defined by the straight line connecting $\mathrm{S}$ and $\mathrm{T}$. It is only intended for comparison purposes.

We have to note that MinDRD samples the radiation for the $k$ chosen points to find the next step, whereas Surf makes a local decision just knowing the positions of the chargers. On the other hand, Graph has global view of the network.

\footnotetext{
${ }^{2}$ we consider only 6 chargers due to high time complexity of the graph algorithm, as the graph that is created is huge to get the offline solution

${ }^{3}$ In our simulation this parameter is set equal to 10 , since this is the recommended value in the corresponding paper
} 


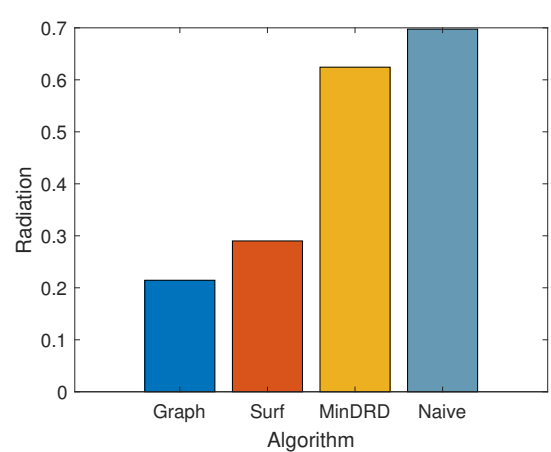

(a) Radiation performance in grid.

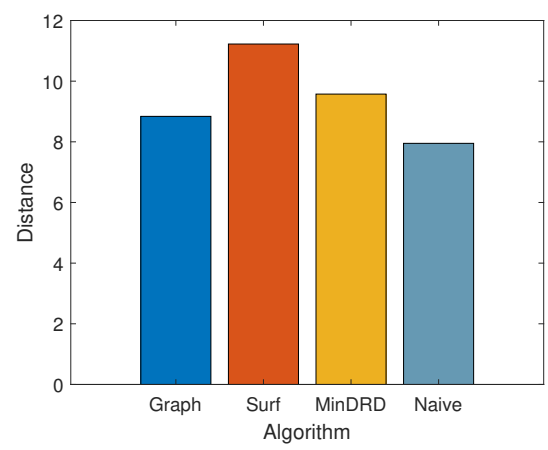

(c) Distance travel performance in grid.

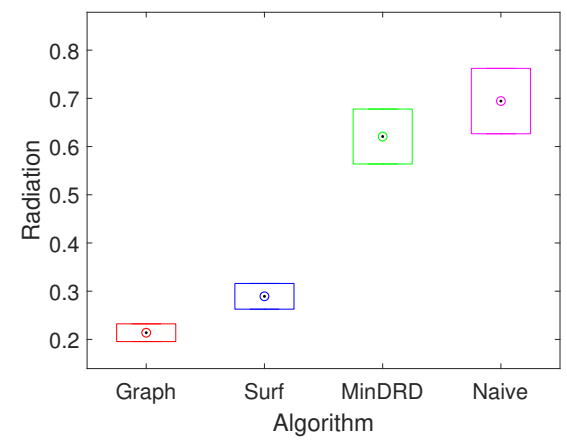

(b) Confidence intervals for radiation performance in grid for 100 repetitions.

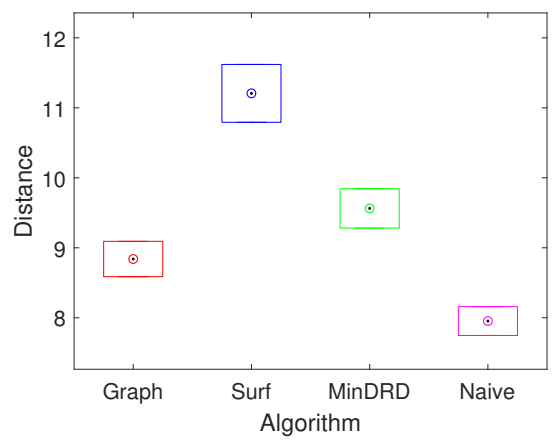

(d) Confidence intervals for distance travel performance in grid for 100 repetitions.

Figure 4. Radiation and distance performance in grid network for 100 repetitions varying starting and target points.

In Figure 3 we present an instance from the grid setup, in which we have colored the paths of the four different approaches. Regarding Surf's path, it begins by having 1 charger in the communication range of the mobile agent, thus it begins by following a circle trajectory until it meets a second charger. Having now two chargers in its communication range, the agent rides the hyperbola of those two, and so on, following the suggestions of best pair function.

Figures $4 \mathrm{a}$ and $4 \mathrm{c}$ depict the radiation and distance metrics for each approach. As we see, Surf outperforms MinDRD and the naive approach (less than half radiation), while at the same time has similar radiation levels with the offline Graph algorithm. Considering the distance metric it becomes obvious the trade off we need to pay to get low radiation levels, which is within acceptable limits. In particular, Graph achieves the smallest radiation while at the same time it travels a distance which is very close to the straight line as the naive approach. In Figures $4 \mathrm{~b}, 4 \mathrm{~d}$ are provided the corresponding confidence intervals which prove a high concentration around the mean and without overlaps between them.

Regarding random network topologies, the simulation verifies the good performance of Surf in radiation (almost half from MinDRD and naive) and the distance cost we need to pay to achieve it (Figures 5a, 5c). The corresponding confidence intervals are sufficient for this case too, and are shown in Figures 5b, 5d. Note that, although in Surf the agent travels slighty longer, which means that it is exposed for longer time to radiation, it manages to keep the radiation low both in random and grid topologies, proving that indeed we manage to find a good path.

Finally, regarding the naive and MinDRD algorithms, the first offers the quickest path but in high radiation as expected, while the latter has a worse distance than naive (still better than Surf), but worse radiation performance. This is due to the algorithm's design which aims to avoid moving near chargers, with respect to the scalar model and the uniform power distribution in the field which depends only on the relative distance of the chargers.

\section{CONCLUSION AND FUTURE WORK}

In this paper we applied the vector model in $E M R$ context for the first time and studied The Minimum Radiation Path Problem in wireless and WPT networks, as both make use of electromagnetic RF sources. We presented two different approaches and an extended numerical simulation, comparing other state-of-the-art approaches with ours. The above validation process was conducted in both grid and random topologies, revealing advantages and weaknesses of each solution per case.

In future we opt to investigate more EMR problems and explore further the vector model and its potential, regarding the EMR concept. Finally, we also wish to add more restrictions 


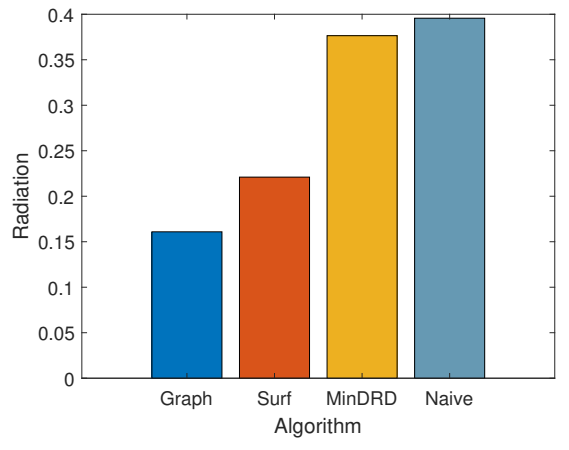

(a) Radiation performance in random networks.

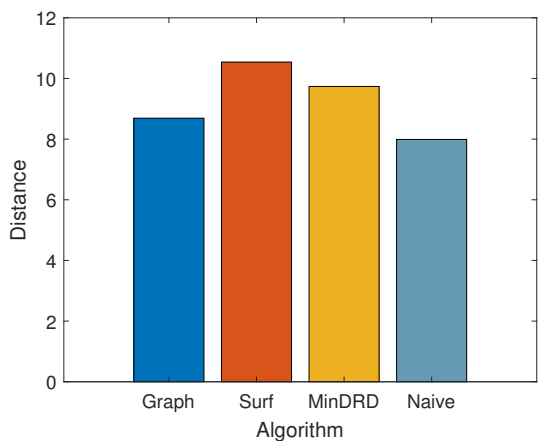

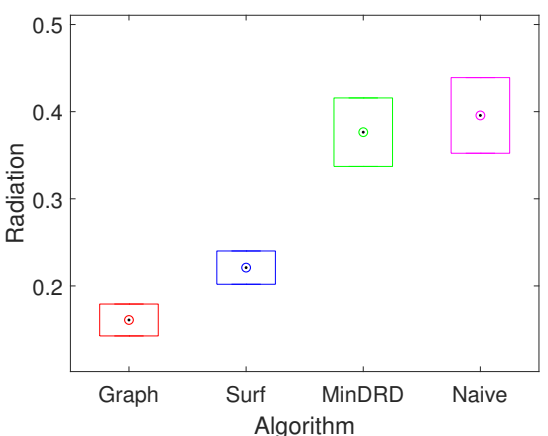

(b) Radiation performance in random networks.

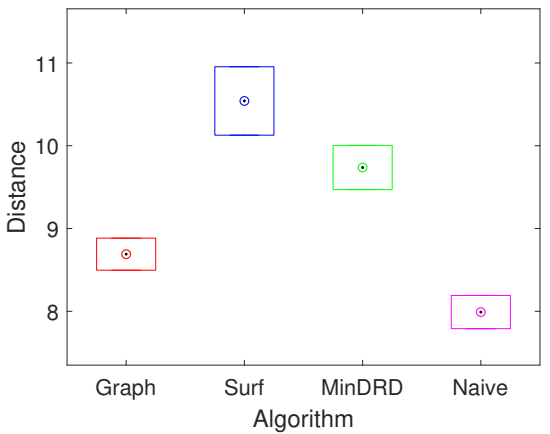

(c) Distance travel performance in random networks. (d) Distance travel performance in random networks.

Figure 5. Radiation and distance performance in random networks for 100 repetitions varying chargers deployment.

and parameters in our approach and solve problems that also address efficient charging alongside EMR safety.

\section{ACKNOWLEDGMENT}

This work was supported by the General Secretariat for Research and Technology (GSRT), the Hellenic Foundation for Research and Innovation (HFRI) and the EU Horizon 2020 Safestrip project.

\section{REFERENCES}

[1] Ioannis Katsidimas, Sotiris Nikoletseas, Theofanis P. Raptis, and Christoforos Raptopoulos. 2017. Efficient Algorithms for Power Maximization in the Vector Model for Wireless Energy Transfer. In Proceedings of the 18th International Conference on Distributed Computing and Networking (ICDCN '17). ACM, New York, NY, USA, Article 30, 10 pages.

[2] M. Y. Naderi, K. R. Chowdhury and S. Basagni, "Wireless sensor networks with RF energy harvesting: Energy models and analysis," 2015 IEEE Wireless Communications and Networking Conference (WCNC), New Orleans, LA, 2015, pp. 1494-1499.

[3] H. Dai, Y. Liu, G. Chen, X. Wu and T. He, "SCAPE: Safe Charging with Adjustable PowEr," 2014 IEEE Conference on Computer Communications Workshops (INFOCOM WKSHPS), Toronto, ON, 2014, pp. 203-204.

[4] L. Li, H. Dai, G. Chen, J. Zheng, Y. Zhao and P. Zeng, "Radiation Constrained Fair Wireless Charging," 2017 14th Annual IEEE International Conference on Sensing, Communication, and Networking (SECON), San Diego, CA, 2017, pp. 1-9.

[5] H. Dai, Y. Liu, A. X. Liu, L. Kong, G. Chen and T. He, "Radiation constrained wireless charger placement," IEEE INFOCOM 2016 - The 35th Annual IEEE International Conference on Computer Communications, San Francisco, CA, 2016, pp. 1-9.

[6] Haipeng Dai, Huizhen Ma, Alex X. Liu, and Guihai Chen. 2018. Radiation Constrained Scheduling of Wireless Charging Tasks. IEEE/ACM Trans. Netw. 26, 1 (February 2018), 314-327.
[7] S. Nikoletseas, T. P. Raptis and C. Raptopoulos, "Low Radiation Efficient Wireless Energy Transfer in Wireless Distributed Systems," 2015 IEEE 35th International Conference on Distributed Computing Systems, Columbus, OH, 2015, pp. 196-204.

[8] S. Nikoletseas, D. Patroumpa, V. K. Prasanna, C. Raptopoulos and J. Rolim, "Radiation Awareness in Three-Dimensional Wireless Sensor Networks," 2012 IEEE 8th International Conference on Distributed Computing in Sensor Systems, Hangzhou, 2012, pp. 176-185.

[9] Constantinos Marios Angelopoulos, Sotiris Nikoletseas, Dimitra Patroumpa, and Christoforos Raptopoulos. 2012. Radiation-aware data propagation in wireless sensor networks. In Proceedings of the 10th ACM international symposium on Mobility management and wireless access (MobiWac '12). ACM, New York, NY, USA, 11-18.

[10] Constantinos Marios Angelopoulos, Julia Buwaya, Orestis Evangelatos, and José Rolim. 2015. Traversal Strategies for Wireless Power Transfer in Mobile Ad-Hoc Networks. In Proceedings of the 18th ACM International Conference on Modeling, Analysis and Simulation of Wireless and Mobile Systems (MSWiM '15). ACM, New York, NY, USA, 31-40.

[11] S. Loyka and V. Mordachev, "Electromagnetic interference and safety in wireless networks: A statistical approach," 2011 5th International Symposium on Medical Information and Communication Technology, Montreux, 2011, pp. 24-28.

[12] K. S. Yildirim, R. Carli and L. Schenato, "Safe Distributed Control of Wireless Power Transfer Networks," in IEEE Internet of Things Journal.

[13] C. J. v. Leeuwen, K. S. Yildirim and P. Pawelczak, "Self Adaptive Safe Provisioning of Wireless Power Using DCOPs," 2017 IEEE 11th International Conference on Self-Adaptive and Self-Organizing Systems (SASO), Tucson, AZ, 2017, pp. 71-80. 